\title{
Primary Malignant Orbital Tumors
}

\author{
Jacquelyn Laplant ${ }^{1}$ Kimberly Cockerham ${ }^{2}$ \\ ${ }^{1}$ Department of Ophthalmology, Tulane University, New Orleans, \\ Louisiana, United States \\ 2 Stanford Department of Ophthalmology, Byers Eye Institute, Palo \\ Alto, California, United States
}

Address for correspondence Kimberly P. Cockerham, MD, Central Valley Eye Medical Group, 36 W Yokuts, Stockton, CA 95207, United States (e-mail: CockerhamMD@gmail.com).

J Neurol Surg B 2021;82:81-90.

\section{Introduction}

A broad spectrum of benign and malignant tumors can involve the orbit and may be primary, secondary to extension from nearby structures, or distant metastasis. The incidence of specific orbital tumors varies significantly in the literature and is heavily dependent on geographic location, referral patterns, and whether only histopathologically confirmed lesions were included. ${ }^{1-3}$ Primary orbital tumors are rare. In a review of 1264 consecutive patients referred to an ocular oncology center, the incidence of cavernous malformations (previously known as cavernous hemangiomas) was reported as $6 \%$ among orbital lesions. ${ }^{1}$ The incidence of malignant primary orbital tumors is even more rare and increases with age due to the higher prevalence of lymphomas and metastasis in the aging population. ${ }^{1,3}$

Primary orbital malignancies are largely classified based on the type of tissue of origin (- Table 1). ${ }^{1,4}$ Lymphoproliferative lesions of the orbit and adnexa are the most common primary malignant orbital tumors in adults, accounting for up to $20 \%$ of all orbital masses. ${ }^{5,6}$ In children, rhabdomyosarcoma (RMS) is the most common primary orbital malignancy
(C) 2021. Thieme. All rights reserved. Georg Thieme Verlag KG, Rüdigerstraße 14, 70469 Stuttgart, Germany
DOI https://doi.org/ 10.1055/s-0040-1722635. ISSN 2193-6331. 
Table 1 Primary malignant orbital tumors

\begin{tabular}{|c|}
\hline Histologic category \\
\hline $\begin{array}{l}\text { Lymphoproliferative } \\
\text { Non-Hodgkin's lymphoma, Hodgkin's lymphoma, Burkitt's } \\
\text { lymphoma }\end{array}$ \\
\hline $\begin{array}{l}\text { Leukemic } \\
\quad \text { Myeloid sarcoma }\end{array}$ \\
\hline $\begin{array}{l}\text { Optic nerve, meningeal } \\
\text { Optic nerve glioma, optic nerve sheath meningioma }\end{array}$ \\
\hline $\begin{array}{l}\text { Peripheral nerve } \\
\text { Malignant peripheral nerve sheath tumor, alveolar } \\
\text { soft-part sarcoma }\end{array}$ \\
\hline $\begin{array}{l}\text { Primary melanocytic } \\
\quad \text { Primary orbital melanoma }\end{array}$ \\
\hline $\begin{array}{l}\text { Myogenic } \\
\text { Rhabdomyosarcoma }\end{array}$ \\
\hline $\begin{array}{l}\text { Lipomatous } \\
\quad \text { Liposarcoma }\end{array}$ \\
\hline $\begin{array}{l}\text { Fibrohistiocytic } \\
\text { Fibrosarcoma, malignant fibrous histiocytoma }\end{array}$ \\
\hline $\begin{array}{l}\text { Osseous } \\
\quad \text { Osteosarcoma, chondrosarcoma, Ewing's sarcoma }\end{array}$ \\
\hline $\begin{array}{l}\text { Lacrimal gland } \\
\text { Epithelial } \\
\text { Adenoid cystic carcinoma, malignant mixed tumor, } \\
\text { mucoepidermoid carcinoma, adenocarcinoma } \\
\text { Nonepithelial } \\
\text { Lymphoma, plasmacytoma }\end{array}$ \\
\hline $\begin{array}{l}\text { Vascular: } \\
\text { Hemangiopericytoma, angiosarcoma, vascular } \\
\text { leiomyosarcoma }\end{array}$ \\
\hline
\end{tabular}

Source: Data from Blandford AD, Perry JD. Classification of orbital tumors. In: Hwang C], Patel BC, Singh AD, eds. Clinical Ophthalmic Oncology: Orbital Tumors. Cham, Switzerland: Springer; 2019:9-15.

accounting for $\sim 40 \%$ of pediatric orbital tumors in the United States. ${ }^{7}$ Malignant tumors of vascular, neural, meningeal, fibrous, and osseous origin can also arise as primary orbital tumors but are rare. ${ }^{7-10}$

Surgery remains an important diagnostic and treatment modality for orbital tumors. For suspected malignant lesions, a biopsy is often performed for histopathologic confirmation prior to more definitive management. The choice of surgical approach is heavily dependent on the location of the neoplasm, its proximity to critical structures, and the goal of the surgery. Advances in endoscopy, microsurgical techniques, and intraoperative image guidance have led the trend toward less invasive orbital surgery with lower morbidity and decreased recovery times. ${ }^{11,12}$ Further, the advent of multimodal therapy has significantly increased survival rates for many malignancies. ${ }^{13,14}$ In this article, the clinical evaluation, surgical approaches, and management of the most commonly encountered primary malignant orbital tumors are discussed.
Table 2 Primary malignant orbital tumor location

\begin{tabular}{|l|l|}
\hline Intraconal & Extraconal \\
\hline Optic nerve glioma ( \pm intra- & Adenoid cystic carcinoma \\
cranial) & Hemangiopericytoma \\
Optic nerve sheath meningi- & Rhabdomyosarcoma \\
oma ( \pm intracranial) & Lacrimal gland lymphoma \\
Malignant nerve sheath tu- & \\
mor & \\
Primary orbital melanoma & \\
Rhabdomyosarcoma & \\
Lymphoma & \\
\hline
\end{tabular}

Sources: Data from Tailor et al ${ }^{18}$ and Karcioglu. ${ }^{19}$

\section{Pearls and Tips}

- For orbital and adnexal lymphoma (OAL) that is associated with systemic disease or of an aggressive form such as diffuse large B cell lymphoma, chemotherapy and immunomodulation are given in addition to localized radiotherapy.

- Intraoperative image guidance can assist in surgical planning, minimize complications, and improve tumor margin clearance. A multidisciplinary surgical team helps prevent damage to critical structures in tumors that traverse orbital and intracranial spaces.

- Incidental orbital tumors can be identified on head scans done for headaches or trauma. The differential diagnoses of a well-circumscribed orbital mass are primarily benign processes: cavernous hemangioma, solitary fibrous tumor, fibroma, and schwannoma. Without biopsy, however, malignancy cannot be excluded.

\section{Clinical Evaluation}

A comprehensive history and orbital examination are essential for directing further studies and distinguishing orbital malignancies from infection or inflammatory orbital disorders. It is important to define the onset and duration of symptoms. A history of systemic disease, malignancy, or surgery should be elicited.

\section{Clinical Presentation}

The clinical presentation of primary orbital malignancies often reflects the site of the lesion due to the effects on nearby tissues and structures. ${ }^{15,16}$ The most frequent presenting symptom of a space-occupying orbital mass in adults is progressive proptosis. In children, more rapid or sudden-onset proptosis can be seen and is highly suggestive of malignancy. ${ }^{7}$ Direction of globe displacement can help localize the tumor site, such as inferonasal displacement from a lacrimal fossa lesion, and superior displacement from a lesion originating from the maxillary sinus. ${ }^{19}$ In contrast, more extensive lesions involving the extraconal and/or intraconal space such as RMS can present with a generalized proptosis. ${ }^{17}$ 
Other clinical manifestations result from the compressive effect of tumors on nearby structures due to the rigid, confined space of the orbit. As such, orbital tumors can also be classified based on their location within the orbit as intraconal, extraconal, and intracanalicular lesions (-Table 2) ${ }^{18,19}$ Intraconal tumors can exert a mass effect on the globe, optic nerve, and extraocular muscles. These patients tend to present with vision loss, axial proptosis, and diplopia secondary to decreased ocular motility. Extraconal lesions can present similarly; however, vision loss is usually seen later in the course. Intracanalicular tumors, such as optic nerve meningiomas or gliomas, often display minimal or delayed proptosis, but can cause early vision loss with associated optic nerve head atrophy, swelling, and optociliary shunt vessels on fundoscopic examination. ${ }^{17,20}$ In a patient presenting with any of these orbital symptoms, a diagnostic workup with imaging is essential to distinguish orbital malignancies from simulating lesions such as orbital inflammation or infection. ${ }^{21,22}$

\section{Imaging}

Orbital imaging is critical in characterizing a tumor's location, extent, internal characteristics, and involvement of nearby structures. ${ }^{18}$ Imaging with computed tomography (CT), magnetic resonance imaging (MRI), or both is considered gold standard. CT is useful for visualization of bony structures and identification of tumor erosion or hyperostosis of bone, whereas MRI is preferred for imaging soft-tissue structures and apical tumors. ${ }^{21,23-25}$ Ultrasonography can also be useful for anterior-located lesions and in pediatric patients. $^{26}$

Orbital lesions display characteristic patterns on crosssectional imaging, which can aid in the diagnosis. ${ }^{18}$ Benign lesions tend to be well circumscribed, whereas malignant lesions are more irregular and poorly defined; however, this does not always hold true. ${ }^{27}$ Importantly, orbital imaging helps identify the best approach for biopsy and other surgical interventions. Intraoperative image guidance with $\mathrm{CT}$ and/or MRI can help in localizing lesions for biopsy, avoiding crucial structures, and achieving tumor-free margins. ${ }^{28,29}$

\section{Biopsy}

Orbital biopsy is often performed to establish a histopathologic diagnosis and formulate a treatment plan. In general, well-defined lesions that are not associated with crucial structures are managed with excisional biopsy. ${ }^{30}$ Incisional biopsy involves partial tissue excision and is indicated for lesions that are infiltrative, straddle surgical boundaries, or pose an unacceptable risk to critical structures. ${ }^{31}$ It is important to obtain adequate superficial, midzonal, and deep tissue for histopathologic examination, especially for suspected lymphoma, and to handle the specimen properly. Fine-needle aspiration biopsy (FNAB) is useful for select cases such as confirmation of metastasis in a patient with a known neoplasm. ${ }^{30}$ Otherwise FNAB is rarely used for suspected malignant lesions due to inadequate tissue sampling and the potential for tumor seeding or tissue injury.

\section{Surgical Approach}

Determining the optimal surgical approach for biopsy, excision, or debulking of primary orbital malignancies is crucial to avoid damage to vital structures. ${ }^{31,32}$ Choice of surgical approach is largely determined by the location of the lesion, and its proximity to orbital structures such as the optic nerve and extraocular muscles. ${ }^{33}$ Advances in endoscopy, microsurgical techniques, and image guidance have increased access to deeper medial and posterior lesions using less invasive approaches. Using a combination of transorbital, transcranial, and endonasal approaches, 360 degrees of access to the orbit is possible without crossing planes of the optic nerve. ${ }^{33-36}$

\section{Histopathologic Diagnosis}

Final diagnosis is made based on the histopathologic analysis. This guides further management, including the need for additional surgery, chemotherapy, and/or radiation. A variety of orbital malignancies can be treated with chemotherapy alone such as lymphoma or a granulocytic sarcoma. ${ }^{21}$ In contrast, RMS and adenoid cystic carcinomas (ACCs) often require multimodal therapy and a multidisciplinary surgical team consisting of an orbital surgeon, neurosurgeon, or otolaryngologist. ${ }^{14,37}$ In the following sections, we describe the most common primary orbital malignancies and current medical and surgical management with representative cases.

\section{Orbital and Adnexal Lymphoma}

Lymphoproliferative disorders are the most common primary orbital malignancies in adults and can affect all parts of the orbit. $^{38}$ Lymphoproliferative disease is considered a spectrum that includes lymphoid hyperplasia, atypical lymphoid hyperplasia, and orbital and adnexal lymphoma (OAL), which all display unpredictable behavior. Malignant lymphoma is the most common lesion, accounting for 70 to $90 \%$ of orbital lymphoproliferative tumors in adults older than 60 years. ${ }^{39}$ OAL can be classified by the type, site involved, and the presence or absence of systemic disease. The majority of OALs are primary tumors of the non-Hodgkin's B cell type. Localized orbital disease is present in 60 to $80 \%$ of cases, with the orbit and adnexa representing the most commonly affected sites, whereas the remaining cases have evidence of systemic disease at presentation. ${ }^{40}$ Hatef et al reported higher rates of systemic involvement at diagnosis in over $50 \%$ of patients with OAL. ${ }^{41}$

Lymphoma can also involve the intraocular tissue and can be primary with or without central nervous system (CNS) lymphoma or secondary to systemic disease. CNS lymphomas usually metastasize to the eye resulting in infiltration of the retina, vitreous, and/or optic nerve head. Primary intraocular lymphoma (PIOL) is considered a subset of primary central nervous system lymphoma (PCNSL) and is distinct from systemic lymphoma. Compared with OAL, PIOL is more rare, although the incidence has increased in recent years due to the higher number of immunocompromised or immunodeficient patients and improved diagnostic modalities. $^{42}$ The incidence of PIOL varies in the literature; 


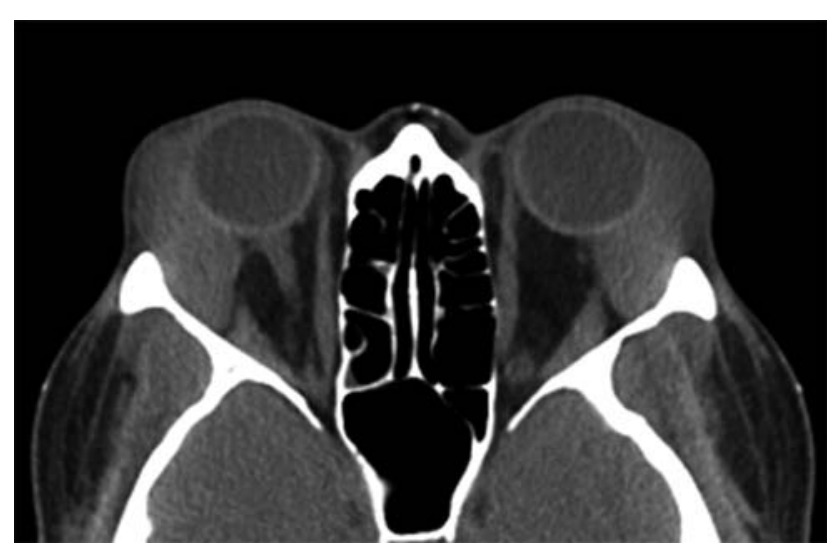

Fig. 1 Lymphoma of the lacrimal gland. Axial orbital computed tomography (CT) scan displays bilateral homogenous lacrimal gland masses molding to the surface of the globes without any bony destructive changes.

however, one nationwide study in Japan estimated it to be $\sim 21$ per 100,000 in patients presenting to multiple referral eye centers. ${ }^{43}$ Diagnosis includes immunohistochemistry, flow cytometry, cytokine, and molecular assessment. Therapeutic options are limited but include methotrexate and radiotherapy to the eye and brain.

OAL typically presents as a progressive, painless mass in the sixth to eighth decades of life. ${ }^{44}$ When in the conjunctiva, it classically presents as a salmon patch. In the orbit, it can involve the lacrimal gland, extraocular muscles, or intra-, and extraconal spaces. ${ }^{39}$ Patients with larger orbital lesions may present with proptosis and diplopia due to extraocular muscle involvement or mass effect, whereas dry eye symptoms are associated with lacrimal gland lesions. Orbital imaging with contrast-enhanced CT or MRI displays characteristic diffuse and ill-defined enhancing lesions with mold-

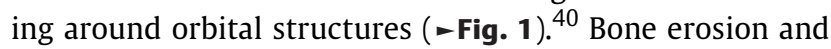
invasion of nearby structures are rare, except in high-grade types such as diffuse large B cell lymphoma. If localized to the orbit, an open biopsy is recommended for diagnosis, whereas a lymph node biopsy can be a less invasive option if systemic disease is present.

Choice of surgical approach is dependent on tumor location. Orbital lymphomas are often anterior and extraconal lesions and can be approached using a transcutaneous or transconjunctival anterior orbitotomy technique according to the quadrant being accessed..$^{45} \mathrm{~A}$ lateral orbitotomy with osteotomy also provides excellent access to lacrimal gland lesions and can access deep-seated lesions that are lateral to the optic nerve and apex. ${ }^{33}$ Following biopsy, initial staging is performed with the help of a medical oncologist to formulate a treatment plan.

Local disease can be successfully treated with radiotherapy, whereas systemic disease is often treated with monoclonal antibody immunotherapy alone (i.e., rituximab) or in combination with chemotherapy. ${ }^{40}$ Complete surgical excision has been reported for highly localized OAL but is not typically a viable option due to the tumor's infiltrative nature. ${ }^{21}$ Goals of treatment are local control and prevention of progression to systemic involvement.

Case 1: A 50-year-old woman with a history of systemic diffuse large B cell lymphoma presented with a 2-month history of progressive proptosis, diplopia, and decreased vision. She demonstrated inferonasal globe displacement, ophthalmoplegia, with periorbital edema and chemosis (-Fig. 2). A left afferent pupillary defect was present and visual acuity was 20/80 and hand motion in the right and left eyes. MRI displayed bilateral lacrimal gland enlargement abutting the lateral and superior rectus muscles with molding around the globe and involvement of the left optic nerve. Histologic findings of the involved left neck lymph node demonstrated diffuse lymphoid infiltrate consisting of large cells with positive staining for CD5, BCL2, and C-MYC. With fluorescence in situ hybridization (FISH) studies, the patient was diagnosed with high-grade B cell lymphoma with MYC and BCL6 rearrangements. She was started on intravenous steroids and R-ICE chemotherapy regimen with significant improvement of her clinical signs and symptoms. Palliative radiation to the orbits was recommended, but the patient passed away before it could be given.

Pearl: For OAL that is associated with systemic disease or of an aggressive form such as diffuse large B cell lymphoma, chemotherapy and immunomodulation are given in addition to localized radiotherapy.

\section{Primary Epithelial Malignancies of the Lacrimal Gland}

Lacrimal gland malignancies can be classified as epithelial and nonepithelial lesions ( - Table 1). In recent studies, 20\% of lacrimal gland lesions are of epithelial origin, of which $45 \%$ are malignant. ${ }^{1,4}$ Of these primary epithelial malignances of the lacrimal gland (PEML), ACC is the most common (60\%), followed by malignant mixed tumor $(20 \%) .{ }^{46}$ Less common malignant lesions include adenocarcinoma and mucoepidermoid carcinoma. ${ }^{47,48}$

Clinical presentation is relatively similar among lacrimal gland tumors, demonstrating a palpable superolateral mass with inferomedial globe displacement, most often in the fourth or fifth decade of life. ${ }^{46}$ Lesions arising in the
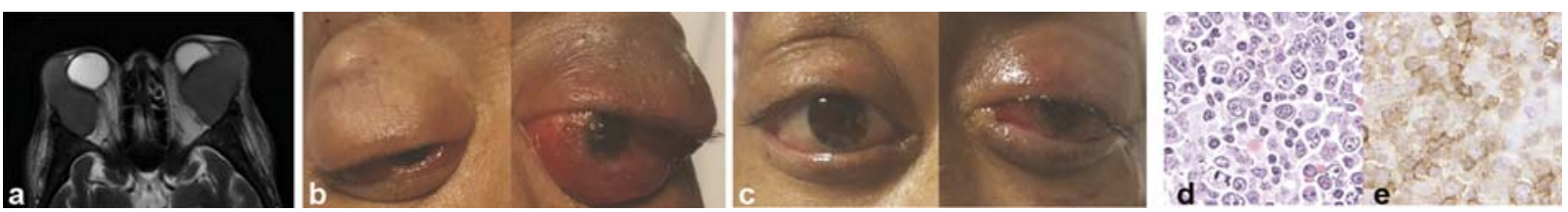

Fig. 2 Diffuse large B cell lymphoma of the lacrimal glands. (a) T2-weighted axial magnetic resonance imaging (MRI) with bilateral lacrimal gland masses with molding around the globes. (b) External photograph of patient on presentation. (c) External photograph demonstrating improvement of proptosis, periorbital edema, and chemosis 2 weeks after induction of R-ICE chemotherapy regimen. (d) Diffuse lymphoid infiltrate consisting of large cells noted on biopsy (H\&E; 40X). (e) Neoplastic cells positive for CD5 (40X). 


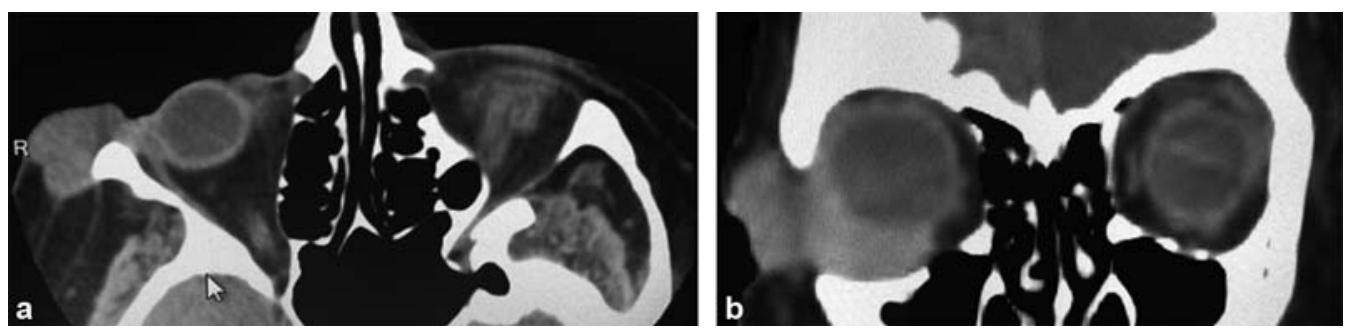

Fig. 3 Primary ductal adenocarcinoma of the lacrimal gland. (a) Axial and (b) coronal orbital computed tomography (CT) scan demonstrates an irregular right orbital mass with ill-defined margins arising from the area of the lacrimal gland.

palpebral lobe can present earlier with abnormal lid contour due to the anterior location compared with orbital lobe lesions. ACC tends to have a more rapid onset and progression. Pain is often seen due to early perineural spread. Malignant lesions also tend to spread posteriorly along the lateral orbital wall and can breach the periosteum or superior orbital fissure later in the disease course. ${ }^{49}$

On CT and MRI, ACC and other PEML are typically described as less defined, elongated soft-tissue masses, possibly with calcification ( - Fig. 3). Lacrimal gland masses can also extend posterolaterally within the constraints of the lateral rectus and lateral orbital wall roof, creating a lateral triangle described as a "wedge sign." ${ }^{49}$ Bone erosion of the orbital roof or lateral wall can be evident even with smaller lesions. ${ }^{46}$ Lesions that involve the skull base via bony erosion, perineural extension, or foraminal spread are associated with a poorer prognosis. ${ }^{50}$ Complete excision should be performed for lesions that appear small, localized, and well circumscribed on imaging to avoid spread of tumors cells. For less defined lesions that mold to the globe, a generous incisional biopsy should be done for histopathologic diagnosis. ${ }^{46}$

Management of ACC varies significantly among institutions. Historically, the most common surgical management is orbital exenteration with removal of affected walls followed by adjuvant radiation due to the high risk of local recurrence and distant metastasis. ${ }^{51,52}$ This requires a multidisciplinary surgical team for radical orbital and skull base surgery to achieve local control. ${ }^{53}$ More recently, there has been a shift toward globe-sparing surgery with adjuvant chemotherapy or radiation. ${ }^{14,54}$ This involves tumor debulking with preservation of the eye and vital orbital structures followed by adjuvant radiation of involved soft tissues and bone including orbital walls, the orbital apex, and cavernous sinus. ${ }^{14}$ Neoadjuvant intra-arterial chemotherapy may also be utilized to shrink the tumor preoperatively. ${ }^{55}$

Mixed malignant tumors are the second most common primary epithelial malignancy of the lacrimal gland and most often arise from preexisting pleomorphic adenomas. Recurrence rates for pleomorphic adenomas vary in the literature but have been shown to be higher after incomplete excision or rupture. Font et $\mathrm{al}^{56}$ reported a 5-year recurrence rate of $3 \%$ for intact excision and 32\% after incomplete excision, whereas Lai et al ${ }^{57}$ found no increased risk of recurrence after incisional biopsy followed by complete excision. However, up to $20 \%$ of recurrent pleomorphic adenomas undergo malignant transformation by 30 years, emphasizing the impor- tance of full excision of suspected pleomorphic adenomas. ${ }^{58,59}$ Management of mixed malignant tumors and other adenocarcinomas of the lacrimal gland are treated in a similar manner to ACC and, depending on size, can be treated by local excision followed by radiotherapy. The prognosis for PEML is determined by cell type, but the prognosis for lacrimal gland carcinomas in general remains poor. ACC is associated with late recurrence even after extensive resections, although additional chemotherapy may improve prognosis. ${ }^{55}$

\section{Rhabdomyosarcoma}

RMS is the most common primary orbital malignancy in children and the most common malignant tumor of childhood to invade the skull base from the orbit. ${ }^{60}$ It often presents in the first or second decade of life with a mean age at diagnosis of 8 years. ${ }^{13}$ RMS is generally classified into embryonal, alveolar, pleomorphic, and botryoid subtypes. Embryonal RMS is the most common subtype, whereas pleomorphic and alveolar forms are associated with the best and worst prognoses, respectively. ${ }^{61}$

Clinical presentation reflects primary tumor location, which is often extraconal and within the anterior or midorbit. ${ }^{62}$ The superonasal orbit is the most common quadrant for RMS. Patients classically present with a rapidly progressive unilateral nonaxial proptosis and periorbital swelling over the course of weeks, which can often resemble infection. Impairment of extraocular motility and optic neuropathy, choroidal folds, and disc edema are common. ${ }^{61}$

Orbital imaging demonstrates a well-circumscribed, irregular, and relatively homogenous mass within orbital soft tissues that enhances moderately with contrast. ${ }^{63}$ Bony destruction and erosion can be seen on larger, aggressive lesions. ${ }^{62}$ Diagnosis is made through open biopsy with or without tumor debulking, to provide adequate tissue for histopathologic evaluation. For smaller anterior lesions, a biopsy can often be achieved through an anterior orbitotomy via a transcutaneous incision or transconjunctival incision depending on location. ${ }^{63} \mathrm{~A}$ comprehensive systemic evaluation is required for suspected orbital RMS with the help of a pediatric oncologist.

Multimodal therapy has significantly reduced the morbidity and mortality of RMS. ${ }^{63,64}$ Current management consists of a combination of surgical biopsy, resection, or debulking followed by systemic chemotherapy and radiation. Complete resection or maximal debulking can be attempted at the initial time of diagnosis but remains controversial due 

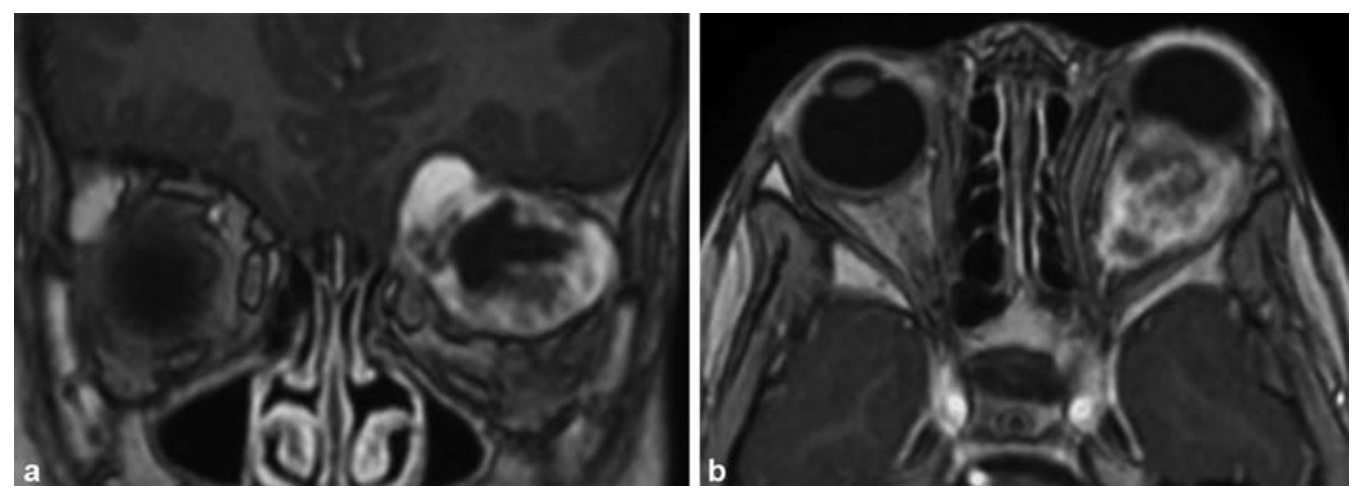

Fig. 4 Parameningeal rhabdomyosarcoma. T1-weighted (a) coronal and (b) axial magnetic resonance imaging (MRI) shows an enhancing irregular retrobulbar mass of the left orbit with dehiscence of the orbital roof and extension into the anterior cranial fossa.

to risk of injury to nearby orbital structures such as the optic nerve. In tumors with intracranial extension or invasion of the paranasal sinuses, a multidisciplinary team is essential to facilitate extraorbital approaches (-Fig. 4). ${ }^{23}$

Risk stratification relies on both a pretreatment (TNM) staging system and the pathologic and surgical grouping system established by the Intergroup rhabdomyosarcoma study (IRS). ${ }^{65}$ The IRS-V study combines groups, stage, and histologic subtype to assign patients to one of four categories and stratify treatment protocols based on risk of recurrence. In the past, histology was thought to serve as the primary prognostic indicator; however, tumor site of origin, nonparameningeal versus parameningeal, has been suggested to better correlate with prognosis. Parameningeal sites include the nasopharynx and nasal cavities, paranasal sinuses, infratemporal and pterygopalatine fossa, and retropharyngeal space. Nonparameningeal RMS includes other sites in the head and neck and is more amenable to complete surgical resection and associated with a better prognosis.

Localized orbital RMS carries the most favorable prognosis with an overall survival rate of greater than $90 \% .{ }^{65}$ Large clinical trials have demonstrated the importance of anatomical site on overall survival rates of pediatric RMS, although differences in treatment approach and outcome data have been identified among studies. ${ }^{66-70}$ The International Society of Pediatric Oncology Malignant Mesenchymal Tumor Study-89 (MMT-89) and IRS-IV reported a 5-year overall survival rate of 59 to 65 and 60 to $73 \%$, respectively, depending on age. ${ }^{70}$ As expected, metastatic disease portends a very poor prognosis with 3-year survival rates of $\sim 25$ to $30 \%$ despite multimodal therapy. ${ }^{67}$ The prognosis of adult RMS remains poor with involvement of less favorable anatomical sites and more aggressive disease. ${ }^{71}$

In cases of recurrence or incomplete resection, exenteration with removal of the associated wall is often performed. Multimodal therapy has significantly increased the 5-year survival rates for orbital RMS from $30 \%$ in the 1970 s to $90 \%$ depending on the extent of disease at diagnosis, tumor border, site, age, histopathology, and therapeutic response. ${ }^{13}$

Case 2: A young child presented with a blind, painful eye secondary to relapsed embryonal RMS of the left orbit with intracranial extension after being lost to follow-up after prior excision and adjuvant chemoradiation therapy
(-Fig. 4). The patient underwent tumor debulking and exenteration with neurosurgery and ophthalmology. Frameless stereotactic navigation was used for planning and intraoperative guidance. Gross total resection was achieved, and adjuvant chemotherapy was given after the patient recovered.

Pearl: Intraoperative image guidance can assist in surgical planning, minimize complications, and improve tumor margin clearance. A multidisciplinary surgical team helps prevent damage to critical structures in tumors that traverse orbital and intracranial spaces.

\section{Hemangiopericytoma}

Hemangiopericytomas are rare encapsulated tumors with a predilection for the superior orbit. ${ }^{21}$ They are more recently considered a spectrum of neoplasms along with solitary fibrous tumors and giant cell angiofibromas. Clinical presentation is similar to that of a cavernous hemangioma; however, hemangiopericytomas are often more aggressive and can spread through the orbit and intracranially. ${ }^{21}$

Middle-aged adults typically present with a slowly progressing proptosis with associated pain, diplopia, and vision loss. $^{72}$ Orbital imaging demonstrates an enhancing, welldefined, homogenous lesion. Management is similar to that of a cavernous hemangioma and involves complete excision. This can often be achieved through various transorbital techniques depending on the location of the lesion. Histopathology demonstrates a mixture of spindle-shaped cells and thin-walled blood vessels that branch in a classic staghorn vascular pattern and positive staining for CD34. Although many lesions are benign, there is a high rate of recurrence and risk of malignant transformation and metastasis, emphasizing the importance of complete removal.

Case 3: A 40-year-old woman presented with an incidental finding on an imaging study done for headaches. She had no proptosis, eye symptoms, or signs. A subsequent MRI of the orbits demonstrated a well-circumscribed mass in the medial orbit (-Fig. 5). A mediolateral orbitotomy was performed to provide enhanced visibility and removal of the large orbital tumor in the medial quadrant. The lateral bone was removed temporarily to allow retraction of the globe into the space where the bone had been. The histopathology was consistent with hemangiopericytoma including thin- 


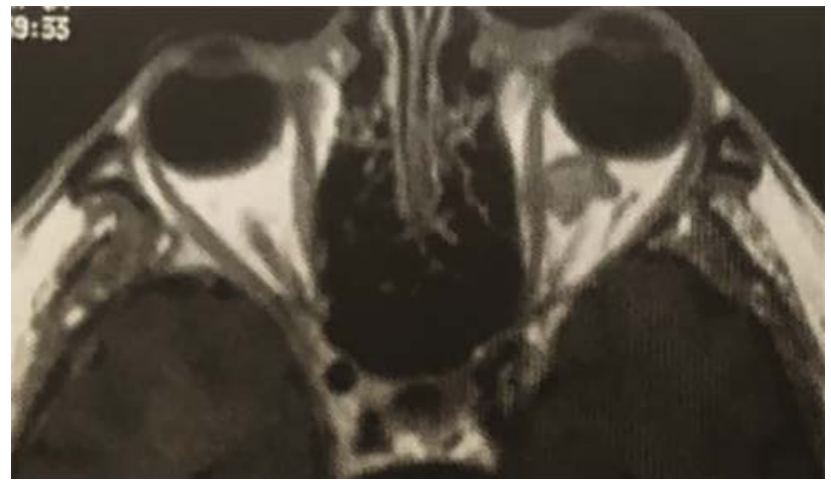

Fig. 5 Axial T2-weighted magnetic resonance imaging (MRI) demonstrates a well-circumscribed homogenous lesion of the medial orbit with heterogenous regions more anteriorly. There are no imaging characteristics that distinguish the very common cavernous hemangioma from the rare hemangiopericytoma with adequate sensitivity and specificity. Histopathology and immunohistochemical staining were consistent with hemangiopericytoma.

walled blood vessels that branch in a staghorn pattern, ovoid or spindle-shaped nuclei, and cells that uniformly stain positively for CD34 and vimentin, Leu-7, factor XIIIa, and HLA-DR. Thirty percent of orbital hemangiopericytomas are histologically compatible with malignancy. This patient's tumor did not demonstrate high mitotic rate, increased cellularity, pleomorphism, necrosis, or hemorrhage, and was negative for the other prognostic markers including Bcl-2, p53, and $\mathrm{K}_{\mathrm{i}}-67$. No adjunctive radiation was performed.

Pearl: Incidental orbital tumors can be identified on head scans done for headaches or trauma. The differential diagnoses of a well-circumscribed orbital mass are primarily benign processes: cavernous hemangioma, solitary fibrous tumor, fibroma, and schwannoma. Without biopsy, however, malignancy cannot be excluded.

\section{Optic Pathway Gliomas}

Optic pathway gliomas (OPG) are the most common tumors of the optic nerve, though they are most commonly of the juvenile pilocytic astrocytoma (JPA) type and exhibit more benign behavior although malignant transformation has been reported. ${ }^{73,74}$ They are the most common intrinsic optic nerve tumors in children and can occur anywhere along the optic pathway. They are a major diagnostic criterion for neurofibromatosis type 1 (NF1). Clinical presentation is dependent on the location of the tumor. The majority of patients experience visual impairment. Proptosis is the presenting symptom in the majority of patients unless localized posterior to the optic chiasm. With CNS invasion, nystagmus, spasmus nutans, seizures, hydrocephalus, and endocrine abnormalities due to hypothalamic involvement can be observed. Optic atrophy and disc edema are often seen on fundoscopic examination. Chiasmal lesions are less likely to demonstrate funduscopic findings but may demonstrate visual field defects. ${ }^{75,76}$

CT and MRI classically demonstrate enlargement of the optic nerve in a fusiform pattern and/or involvement of the optic chiasm. OPG typically appear hypointense on T1- weighted imaging and hyperintense on T2-weighted imaging. Imaging is usually sufficient for diagnosis and biopsy is reserved for atypical lesions.

Management of OPG is challenging due to their unpredictable behavior, although generally, declining growth rate is observed over time. Treatment is dependent on location of the tumor and the patient's symptoms, visual function, and clinical course. Baseline radiographic studies and clinical examination are essential. Conservative management with clinical and radiographic studies is often chosen unless patients demonstrate progressive optic nerve dysfunction, visual loss, proptosis, progressive visual field changes, or evidence of hypothalamic invasion. Management of progressive disease typically consists of chemotherapy, or, in select cases, radiotherapy, as an adjunctive or alternative therapy to surgery and can result in stabilization of vision with tumor regression. Surgery is less commonly performed today and limited to select cases such as massive proptosis with corneal exposure. Surgery may also be indicated for lesions invading the optic canal toward the optic chiasm. Patients with NF1 tend to experience less mortality and a better visual prognosis, whereas patients without NF1 and increasing age have a poorer prognosis. In a review of OPG of all locations, 38\% of cases demonstrated tumor recurrence or progression. ${ }^{77}$

\section{Malignant Optic Nerve Glioma}

Malignant optic nerve gliomas are rare and often classified as anaplastic astrocytoma or glioblastoma multiforme. ${ }^{76}$ These lesions typically present in middle age although this varies significantly. Clinical presentation is dependent on the portion of the optic nerve involved but typically affects the optic chiasm. Patients present with acute onset of visual impairment. An afferent pupillary defect, optic nerve edema, or atrophy can be seen and complicate diagnosis. Proptosis is minimal or absent. Involvement or spread of the tumor from or to the optic chiasm can present with temporal visual field defects followed by rapid involvement of the other optic nerve. $^{77}$

On imaging, enlargement and enhancement of the optic chiasm and/or the optic nerves is seen and can be misdiagnosed as optic neuritis. Management consists of biopsy or wide surgical excision when feasible. To access the lesion, a transcranial approach is often required. Treatment with radiation is often considered palliative, whereas combination with chemotherapy can mildly extend survival. Unfortunately, even with multimodal therapy, prognosis is poor and the tumor is usually fatal within months. $^{78}$

\section{Malignant Peripheral Nerve Sheath Tumors}

Malignant peripheral nerve sheath tumor (MPNST) is another rare malignant orbital tumor and may develop de novo, after radiation, or, most commonly, in patients with NF1. ${ }^{79}$ The clinical presentation for MPNST is relatively nonspecific. Patients may present with proptosis and downward displacement of the globe in the fourth and fifth decades, or earlier in patients with NF1. It is commonly associated with pain, hypoesthesia, paresthesia, and vision loss. ${ }^{10}$ 
CT can demonstrate a well-circumscribed or irregular, poorly defined mass that most commonly affects the supraorbital nerve. ${ }^{80}$ It is often associated with bone destruction, and enlargement of the superior orbital fissure can be seen. ${ }^{10}$ Once diagnosed, an orbital MPNST is treated with wide excision and often necessitates exenteration with removal of adjacent bone followed by radiation or chemotherapy. ${ }^{30}$ MPNST is associated with a poor prognosis and recurrence is common with direct intracranial extension and distant metastasis. ${ }^{81}$

\section{Conclusion}

Primary orbital malignancies can have variable manifestations and treatment indications depending on the pathology. Clinical presentation is essential in forming a differential and guiding further investigations. Advances in orbital imaging have revolutionized the diagnosis of orbital tumors and, when combined with orbital examination, can often lead to a diagnosis without biopsy. When biopsy is indicated, choice of surgical approach is critical in avoiding injury to orbital structures while obtaining adequate tissue.

Multidisciplinary teams have facilitated newer, less invasive surgical approaches to the orbit using endoscopy and combined transorbital and transcranial approaches. Multimodal therapy has further improved the treatment of certain orbital malignancies that previously carried a poor prognosis and can permit globe-sparing surgery.

\section{Conflict of Interest}

None declared.

\section{References}

1 Shields JA, Shields CL, Scartozzi R. Survey of 1264 patients with orbital tumors and simulating lesions: The 2002 Montgomery Lecture, part 1. Ophthalmology 2004;111(05):997-1008

2 Margo CE, Mulla ZD. Malignant tumors of the orbit. Analysis of the Florida Cancer Registry. Ophthalmology 1998;105(01):185-190

3 Bonavolontà G, Strianese D, Grassi P, et al. An analysis of 2,480 space-occupying lesions of the orbit from 1976 to 2011. Ophthal Plast Reconstr Surg 2013;29(02):79-86

4 Shields JA, Bakewell B, Augsburger JJ, Flanagan JC. Classification and incidence of space-occupying lesions of the orbit. A survey of 645 biopsies. Arch Ophthalmol 1984;102(11):1606-1611

5 Rootman J, White V, Connors J, Gascoyne R. Lymphoproliferative, leukemic, and histiocytic lesions of the orbit. In: Rootman J, ed. Diseases of the Orbit: A Multidisciplinary Approach. 2nd ed Philadelphia, PA: Lippincott Williams \& Wilkins; 2003:385-416

6 Pastore G, Peris-Bonet R, Carli M, Martínez-García C, Sánchez de Toledo J, Steliarova-Foucher E. Childhood soft tissue sarcomas incidence and survival in European children (1978-1997): report from the Automated Childhood Cancer Information System project. Eur J Cancer 2006;42(13):2136-2149

7 Karcioglu ZA, Hadjistilianou D. Malignant pediatric tumors. In: Karcioglu ZA, ed. Orbital Tumors: Diagnosis and Treatment. New York, NY: Springer; 2015:349-359

8 Mirzai H, Baser EF, Tansug N, Nese N, Isisag A. Primary orbital neuroblastoma in a neonate. Indian J Ophthalmol 2006;54(03): 206-208

9 Guzowski M, Tumuluri K, Walker DM, Maloof A. Primary orbital Ewing sarcoma in a middle-aged man. Ophthal Plast Reconstr Surg 2005;21(06):449-451
10 Patel BC. Malignant orbital tumors. In: Hwang CJ, Patel BC, Singh AD, eds. Clinical Ophthalmic Oncology: Orbital Tumors. Berlin: Springer; 2019:201-215

11 Al-Qudah M. Image-guided sinus surgery in sinonasal pathologies with skull base/orbital erosion. J Craniofac Surg 2015;26(05): 1606-1608

12 Felippu A, Mora R, Guastini L, Peretti G. Transnasal approach to the orbital apex and cavernous sinus. Ann Otol Rhinol Laryngol 2013;122(04):254-262

13 Shields CL, Shields JA, Honavar SG, Demirci H. Clinical spectrum of primary ophthalmic rhabdomyosarcoma. Ophthalmology 2001; 108(12):2284-2292

14 Esmaeli B, Yin VT, Hanna EY, et al. Eye-sparing multidisciplinary approach for the management of lacrimal gland carcinoma. Head Neck 2016;38(08):1258-1262

15 Bejjani GK, Cockerham K. Diagnosis and management of orbital tumors. Contemp Neurosurg 2003;25(02):1-8

16 Kennerdell JS, Cockerham K. Orbital diagnosis. In: Nesi FA, ed. Smith's Textbook of Ophthalmic Plastic and Reconstructive Surgery. 2nd ed. St Louis, MO: Mosby; 1997:705-712

17 Karcioglu ZA. Orbital Tumors: Diagnosis and Treatment. New York, NY: Springer; 2014

18 Tailor TD, Gupta D, Dalley RW, Keene CD, Anzai Y. Orbital neoplasms in adults: clinical, radiologic, and pathologic review. Radiographics 2013;33(06):1739-1758

19 Karcioglu ZA. Surgical techniques for orbital tumors. In: Karcioglu ZA, ed. Surgical Ophthalmic Oncology. Cham, Switzerland: Springer; 2019:117-127

20 Cockerham K. Meningiomas and sarcomas. In: Miller NR, ed. Walsh and Hoyt's Clinical Neuro-Ophthalmology. Philadelphia, PA: Lippincott Williams \& Wilkins; 1997:2017-2082

21 Shinder R, Esmaeli B. Primary orbital cancers in adults. In: Esmaeli B, ed. Ophthalmic Oncology. Berlin: Springer; 2010:3-12

22 Cockerham KP, Hong SH, Browne EE. Orbital inflammation. Curr Neurol Neurosci Rep 2003;3(05):401-409

23 Priel A, Oh S-R, Kikkawa DO, Korn BS. Surgical approaches to the orbit and optic nerve. In: Smith and Nesi's Ophthalmic Plastic and Reconstructive Surgery. New York, NY: Springer; 2012:793-805

24 Kennerdell JS, Cockerham K. Computed tomography of the orbit. In: Nesi FA, ed. Smith's Textbook of Ophthalmic Plastic and Reconstructive Surgery. 2nd ed. St. Louis, MO: Mosby; 1997: 778-806

25 Cockerham K, Kennerdell JS. Magnetic resonance imaging of the orbit. In: Nesi FA, ed. Smith's Ophthalmic Plastic and Reconstructive Surgery. 2nd ed. St. Louis, MO: Mosby; 1997:807-827

26 Nasr AM, Chacra GIA. Ultrasonography in orbital differential diagnosis. In: Karcioglu Z, ed. Orbital Tumors. New York, NY: Springer; 2015:69-81

27 Ben Simon GJ, Annunziata CC, Fink J, Villablanca P, McCann JD, Goldberg RA. Rethinking orbital imaging establishing guidelines for interpreting orbital imaging studies and evaluating their predictive value in patients with orbital tumors. Ophthalmology 2005;112(12):2196-2207

28 Campbell AA, Grob SR, Yoon MK. Novel surgical approaches to the orbit. Middle East Afr J Ophthalmol 2015;22(04):435-441

29 Ali MJ, Naik MN, Kaliki S, Dave TV. Interactive navigation-guided ophthalmic plastic surgery: the usefulness of computed tomography angiographic image guidance. Ophthal Plast Reconstr Surg 2016;32(05):393-398

30 Shields JA, Shields CL. Eyelid, Conjunctival, and Orbital Tumors: An Atlas and Textbook. Philadelphia, PA: Lippincott Williams \& Wilkins; 2008

31 Verity DH, Rose GE. Principles of orbital surgery. In: Hwang CJ, Patel BC, Singh AD, eds. Clinical Ophthalmic Oncology: Orbital Tumors. Berlin: Springer; 2019:261-274

32 Bejjani GK, Cockerham K, Maroon JC, Kennerdell JS. Orbital tumors. In: Youmans JR, ed. Youmans Neurologic Surgery. 5th ed. Philadelphia, PA: Saunders; 2004:1371-1381 
33 Paluzzi A, Gardner PA, Fernandez-Miranda JC, et al. "Round-theclock" surgical access to the orbit. J Neurol Surg B Skull Base 2015; 76(01):12-24

34 Murchison AP, Rosen MR, Evans JJ, Bilyk JR. Endoscopic approach to the orbital apex and periorbital skull base. Laryngoscope 2011; 121(03):463-467

35 Tsirbas A, Kazim M, Close L. Endoscopic approach to orbital apex lesions. Ophthal Plast Reconstr Surg 2005;21(04): 271-275

36 Matsuda M, Akutsu H, Tanaka S, Matsumura A. Combined simultaneous transcranial and endoscopic endonasal resection of sphenoorbital meningioma extending into the sphenoid sinus, pterygopalatine fossa, and infratemporal fossa. Surg Neurol Int 2017;8:185

37 Bejjani GK, Cockerham KP, Kennerdel JS, Maroon JC. A reappraisal of surgery for orbital tumors. Part I: extraorbital approaches. Neurosurg Focus 2001;10(05):E2

38 Demirci H, Shields CL, Shields JA, Honavar SG, Mercado GJ, Tovilla JC. Orbital tumors in the older adult population. Ophthalmology 2002;109(02):243-248

39 Demirci H, Shields CL, Karatza EC, Shields JA. Orbital lymphoproliferative tumors: analysis of clinical features and systemic involvement in 160 cases. Ophthalmology 2008;115(09): 1626-16311631.e1-1631.e3

40 Aronow ME, Hill BT, Singh AD. Orbital and adnexal lymphoma. In: Perry JD, Singh AD, eds. Clinical Ophthalmic Oncology: Orbital Tumors. Berlin: Springer; 2014:123-139

41 Hatef E, Roberts D, McLaughlin P, Pro B, Esmaeli B. Prevalence and nature of systemic involvement and stage at initial examination in patients with orbital and ocular adnexal lymphoma. Arch Ophthalmol 2007;125(12):1663-1667

42 Mulay K, Narula R, Honavar SG. Primary vitreoretinal lymphoma. Indian J Ophthalmol 2015;63(03):180-186

43 Mochizuki M, Singh AD. Epidemiology and clinical features of intraocular lymphoma. Ocul Immunol Inflamm 2009;17(02): 69-72

44 White WL, Ferry JA, Harris NL, Grove AS Jr. Ocular adnexal lymphoma. A clinicopathologic study with identification of lymphomas of mucosa-associated lymphoid tissue type. Ophthalmology 1995;102(12):1994-2006

45 Cockerham KP, Bejjani GK, Kennerdell JS, Maroon JC. Surgery for orbital tumors. Part II: transorbital approaches. Neurosurg Focus 2001;10(05):E3

46 Shields JA, Shields CL, Epstein JA, Scartozzi R, Eagle RC Jr. Review: primary epithelial malignancies of the lacrimal gland: the 2003 Ramon L. Font lecture. Ophthal Plast Reconstr Surg 2004;20(01): $10-21$

47 Milman T, Shields JA, Husson M, Marr BP, Shields CL, Eagle RC Jr. Primary ductal adenocarcinoma of the lacrimal gland. Ophthalmology 2005;112(11):2048-2051

48 Verity DH, Rose GE. Lacrimal gland tumors. In: Hwang CJ, Patel BC, Singh AD, eds. Clinical Ophthalmic Oncology: Orbital Tumors. Berlin: Springer; 2019:165-175

49 Lorenzano D, Rose GE. The "wedge sign": an imaging sign for aggressive lacrimal gland disease. Ophthalmology 2017;124(07): 1081-1083

50 Ramakrishna R, Raza SM, Kupferman M, Hanna E, DeMonte F. Adenoid cystic carcinoma of the skull base: results with an aggressive multidisciplinary approach. J Neurosurg 2016;124 (01):115-121

51 Zoumalan CI, Zoumalan RA, Cockerham KP. Surgical management of lacrimal gland tumors. Oper Tech Otolaryngol Head Neck Surg 2008;19(04):252-257

52 Abugo UE, Cockerham K. Exenteration. In: Levine MR, ed. Manual of Oculoplastic Surgery. Berlin: Springer; 2018

53 Esmaeli B, Golio D, Kies M, DeMonte F. Surgical management of locally advanced adenoid cystic carcinoma of the lacrimal gland. Ophthal Plast Reconstr Surg 2006;22(05):366-370
54 Ahmad SM, Esmaeli B, Williams M, et al. American Joint Committee on Cancer classification predicts outcome of patients with lacrimal gland adenoid cystic carcinoma. Ophthalmology 2009; 116(06):1210-1215

55 Tse DT, Kossler AL, Feuer WJ, Benedetto PW. Long-term outcomes of neoadjuvant intra-arterial cytoreductive chemotherapy for lacrimal gland adenoid cystic carcinoma. Ophthalmology 2013; 120(07):1313-1323

56 Font RL, Gamel JW, Jacobiec FA. Epithelial tumors of the lacrimal gland: an analysis of 265 cases. In: Jakobiec FA, ed. Ocular and Adnexal Tumors. Birmingham, AL: Aesculapius Publishing; 1978: 787-805

57 Lai T, Prabhakaran VC, Malhotra R, Selva D. Pleomorphic adenoma of the lacrimal gland: is there a role for biopsy? Eye (Lond) 2009; 23(01):2-6

58 Currie ZI, Rose GE. Long-term risk of recurrence after intact excision of pleomorphic adenomas of the lacrimal gland. Arch Ophthalmol 2007;125(12):1643-1646

59 Vagefi MR, Hong JE, Zwick OM, Bedrossian EH Jr, Seiff SR, Cockerham KP. Atypical presentations of pleomorphic adenoma of the lacrimal gland. Ophthal Plast Reconstr Surg 2007;23(04): 272-274

60 Ammirati M, Zarzour H. Overview of skull base tumors. In: Winn HR, ed. Youmans and Winn Neurological Surgery. Philadelphia, PA: Saunders Elsevier; 2017:1222-1242

61 Perry JD, Patel BC. Orbital rhabdomyosarcoma. In: Hwang CJ, Patel BC, Singh AD, eds. Clinical Ophthalmic Oncology: Orbital Tumors. Berlin: Springer; 2019:217-229

62 Chung EM, Smirniotopoulos JG, Specht CS, Schroeder JW, Cube R. From the archives of the AFIP: pediatric orbit tumors and tumorlike lesions: nonosseous lesions of the extraocular orbit. Radiographics 2007;27(06):1777-1799

63 Katowitz WR, Fries PD. Malignant pediatric orbital tumors. In: Katowitz JA, Katowitz WR, eds. Pediatric Oculoplastic Surgery. Berlin: Springer; 2018:743-770

64 Crist W, Gehan EA, Ragab AH, et al. The Third Intergroup Rhabdomyosarcoma Study. J Clin Oncol 1995;13(03):610-630

65 Raney RB, Maurer HM, Anderson JR, et al. The Intergroup rhabdomyosarcoma study group (IRSG): major lessons from the IRS-I through IRS-IV studies as background for the current IRS-V treatment protocols. Sarcoma 2001;5(01):9-15

66 Malempati S, Hawkins DS. Rhabdomyosarcoma: review of the Children's Oncology Group (COG) Soft-Tissue Sarcoma Committee experience and rationale for current COG studies. Pediatr Blood Cancer 2012;59(01):5-10

67 Hawkins DS, Spunt SL, Skapek SXCOG Soft Tissue Sarcoma Committee. Children's Oncology Group's 2013 blueprint for research: Soft tissue sarcomas. Pediatr Blood Cancer 2013;60(06): 1001-1008

68 Reilly BK, Kim A, Peña MT, et al. Rhabdomyosarcoma of the head and neck in children: review and update. Int J Pediatr Otorhinolaryngol 2015;79(09):1477-1483

69 Turner JH, Richmon JD. Head and neck rhabdomyosarcoma: a critical analysis of population-based incidence and survival data. Otolaryngol Head Neck Surg 2011;145(06):967-973

70 Donaldson SS, Anderson JR. Rhabdomyosarcoma: many similarities, a few philosophical differences. J Clin Oncol 2005;23(12): 2586-2587

71 Bompas E, Campion L, Italiano A, et al. Outcome of 449 adult patients with rhabdomyosarcoma: an observational ambispective nationwide study. Cancer Med 2018;7(08):4023-4035

72 Bernardini FP, de Conciliis C, Schneider S, Kersten RC, Kulwin DR. Solitary fibrous tumor of the orbit: is it rare? Report of a case series and review of the literature. Ophthalmology 2003;110(07): 1442-1448

73 Wilson WB, Feinsod M, Hoyt WF, Nielsen SL. Malignant evolution of childhood chiasmal pilocytic astrocytoma. Neurology 1976;26 (04):322-325 
74 de Keizer RJW, de Wolff-Rouendaal D, Bots GTA, Thomeer RT, Brouwer OF, Vielvoye GJ. Optic glioma with intraocular tumor and seeding in a child with neurofibromatosis. Am J Ophthalmol 1989;108(06):717-725

75 Katowitz WR, Fries PD, Kazim M. Benign pediatric orbital tumors. In: Katowitz JA, Katowitz WR, eds. Pediatric Oculoplastic Surgery. Berlin: Springer; 2018:667-741

76 Dutton JJ. Tumors of the optic nerve. In: Hwang CJ, Patel BC, Singh AD, eds. Clinical Ophthalmic Oncology: Orbital Tumors. Berlin: Springer; 2019:137-148

77 Dutton JJ. Gliomas of the anterior visual pathway. Surv Ophthalmol 1994;38(05):427-452

78 Alireza M, Amelot A, Chauvet D, Terrier LM, Lot G, Bekaert O. Poor prognosis and challenging treatment of optic nerve malignant gliomas: literature review and case report series. World Neurosurg 2017;97:751 e751-751 e756

79 Minovi A, Basten O, Hunter B, Draf W, Bockmühl U. Malignant peripheral nerve sheath tumors of the head and neck: management of 10 cases and literature review. Head Neck 2007;29(05): 439-445

80 Lyons CJ, McNab AA, Garner A, Wright JE. Orbital malignant peripheral nerve sheath tumours. Br J Ophthalmol 1989;73 (09):731-738

81 Erzurum SA, Melen O, Lissner G, et al. Orbital malignant peripheral nerve sheath tumors. Treatment with surgical resection and radiation therapy. J Clin Neuroophthalmol $1993 ; 13(01): 1-7$ 